\title{
Safe Acting and Manipulation in Human Environments: A Key Concept for Robots in our Society
}

\author{
Sami Haddadin, Sven Parusel, Rico Belder, Alin Albu-Schäffer, and Gerd Hirzinger
}

\begin{abstract}
In this paper we review our work on safe acting and manipulation in human environments. In order for a robot to be able to safely interact with its environment it is primary to be able to react to unforeseen events in real-time on basically all levels of abstraction. Having this goal in mind, our contributions reach from fundamental understanding of human injury due to robot-human collisions as the underlying metric for "safe" behavior, various interaction control schemes that ground on the basic components impedance control and collision behavior, to real-time motion planning and behavior based control as an interface level for task planning. A significant amount of this work has found found its way into international standardization committees, products, and was applied in numerous real-world applications.
\end{abstract}

\section{INTRODUCTION}

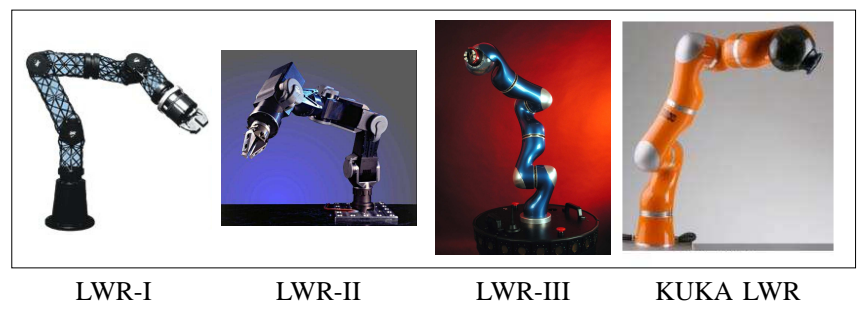

Fig. 1. The generations of DLR light-weight robots (LWR-I, LWR-II, and LWR-III) and the commercialized version (KUKA LWR).

Finally, first robotic systems gained sufficient control capabilities to perform delicate and complex manipulation and physical human-robot interaction (pHRI) tasks that require the dynamic exchange of physical forces between the robot and its environment. The fully torque-controlled DLR Lightweight Robot III (LWR-III) is such a device [1] and was recently commercialized by the robot manufacturer KUKA (KUKA LWR) [2]. This step made it possible to automate difficult and up to now still manually executed assembly tasks. In particular, the achieved sensible and fast manipulation capabilities [3], [4], [5], [6] of the robot prevent damage from the handled potentially fragile objects and humans directly interacting with the device. Recently, there is strong interest in making classical safety barriers, as e.g. fences or light barriers, obsolete for these interactive devices in order to enable direct physical cooperation between human and robot. For understanding the risks of this undertaking we performed a series of safety investigations [7], [8], [9], [10], [11], [12], [13], which led to fundamental insight into the potential injury a human would suffer due to a collision with a robot. Furthermore, we developed human-friendly interaction control and motion schemes that enable the robot

S. Haddadin, S. Parusel, R. Belder, A. Albu-Schäffer, and G. Hirzinger are with the Institute of Robotics and Mechatronics, DLR - German Aerospace Center, Wessling, Germany, contact: sami . haddadin@dlr.de to show sophisticated real-time responses on interaction force level, motion planning, and real-time task planning [14], [4], [15], [16], [17], [18]. Generally, our approach of embodying reactivity on all levels of robot design and control is to our understanding the core to safe acting and manipulation in human environments. Consequently, the careful design and selection of methods that satisfy this requirement was our main premise.

In this paper we give an overview of the developed analysis tools, control schemes, motion planners, and real-time behaviors for robots that are sought to act and manipulate in human environments. We intend to give a "bird's eye" view on the available repertoire of tools and how the developed methodologies, insights, and algorithms impact robotics in general.

\section{Technologies And Methods}

\section{A. Lightweight \& mechatronic robot design}

The most basic step for building robots that interact with dynamic environments is to design them compact, lightweight, and with high payload. Only light structures are capable of appropriate physical reaction to external forces, i.e. have low intrinsic impedance. Secondly, the robot's proprioceptive sensorization is a key element. Apart from standard motor position sensing, joint torque sensing together with accurate flexible joint dynamics modeling enable real torque control and the sensation of contact forces. In this line of thinking we have developed a series of torque controlled lightweight robots at DLR that are suitable for a diverse range of applications involving space, industry, medical, and domestic use. Figure 1 shows the history of the DLR Lightweight robots, resulting in its commercialized version: the KUKA LWR [2]. Apart from minor modifications, this manipulator has exactly the same design as the $3^{\text {rd }}$ generation of the DLR Lightweight robots [1], which are kinematically redundant, $7-\mathrm{DoF}$, joint-torque controlled flexible joint robots. The current version is the result of 15 years of research that produced three consecutive generations. Since the LWR-III weighs $13.5 \mathrm{~kg}$ and is able to handle loads up to $15 \mathrm{~kg}$, an approximate load-to-weight ratio of 1 is achieved ${ }^{1}$. The robot is a modular system and the joints are linked via carbon-fiber structures. The electronic parts, including power converting elements are integrated into the structure of the arm. Each joint is equipped with a motor position and a joint-torque sensor. Additionally, a 6-DoF force sensor can be embedded in the wrist. All electronics, motors, and gears are integrated into the arm, which makes the robot very compact and portable.

${ }^{1}$ Please note that the nominal payload for the KUKA LWR is $7 \mathrm{~kg}$, but it is able to handle up to $15 \mathrm{~kg}$ for research purposes. 


\section{B. Interaction and manipulation control}

Apart from reducing the reflected mechanical impedance of a robot in order to "make the mechanics sensitive", the design of interaction control schemes is an essential step for sensitive force exchange with the environment. The most widely used control approach to physically interact with robots is probably impedance control and its related schemes, introduced in the pioneering work of Neville Hogan [19] and extended to flexible joint robots in [20], [21], [22], [3], [5]. This type of controller imposes a desired physical behavior with respect to external forces on the robot. For instance the robot is controlled to behave like a Cartesian second order mass-spring-damper system, see Fig. 2.

$$
\mathcal{F}_{\text {ext }}=M_{x}\left(\ddot{\mathbf{x}}-\ddot{\mathbf{x}}_{d}\right)+D_{x}\left(\dot{\mathbf{x}}-\dot{\mathbf{x}}_{d}\right)+K_{x}\left(\mathbf{x}-\mathbf{x}_{d}\right),
$$

where $\mathbf{x}, \mathbf{x}_{d} \in \mathbb{R}^{6}$ are the current robot and desired tip position, $\mathcal{F}_{\text {ext }} \in \mathbb{R}^{6}$ is the external wrench and $M_{x}, K_{x}, D_{x} \in \mathbb{R}^{6 \times 6}$ are the desired Cartesian inertia, stiffness, and damping tensors ${ }^{2}$. Consequently, impedance control allows to realize compliance of the robot by means of active control. Interaction with an impedance controlled robot is robust and intuitive, since in addition to the commanded trajectory, a (local) disturbance response is defined. A major advantage of impedance control is that discontinuities like contact-non-contact do not create such stability problems as they occur with for example hybrid force control [23]. However, important open questions still need to be tackled from a control point of view, such as how to automatically and/or adaptively adjust the impedance parameters according to the current task. First work in this direction can be found in [24], [6].

Apart from nominal interaction control, a robot sharing its workspace with humans and physically interact with its environment should be able to quickly detect collisions and safely react to them. In the absence of external sensing, relative motions between robot and environment/human are unpredictable and unexpected collisions may occur at any location along the robot arm. Various algorithms for coping with this problem were developed and evaluated. Efficient collision detection methods that use only proprioceptive robot sensors and provide also directional information for a safe robot reaction after collisions are introduced and validated [14], [4].

Since our collision detection method gives not only binary contact information but an accurate estimation of the external torques, this information can be used to classify the sensed contact according to its severity. Based on this information it is possible to design application specific reaction patterns that are automatically executed if the required stimulus is sensed.

\footnotetext{
${ }^{2}$ Please note that for the LWR-III we leave the inertia unshaped in order to preserve passivity of the controller. In turn, damping design becomes an important issue since the eigenfrequency is due to the Operational space mass matrix position dependent. Details can be found in [3].
}

Basically, a severity mapping $s m: \tau_{\text {ext }} \rightarrow s$ can be designed either as a fixed stimulus type $\rightarrow$ reaction or a rather complex decision algorithm. In particular, this local interpretation of contact can classify the intensity and hardness of the contact based on contact frequencies and force amplitudes. This enables the robot to act locally very quickly, if unexpected interaction forces occur and act according to specified patterns (some details on this are given in Sec. II-E). This can e.g. be used for activating automatic recovery strategies during identified failed grasping of objects, especially for avoiding the risk of damaging them.

The Cartesian impedance controller as well as the collision detection and reaction methods are already integrated in the KUKA LWR, i.e. available as a commercial product. Important to notice is that these novel features are considered as the key to enable safe pHRI by industry.

\section{Injury based safety analysis}

During unexpected collisions with humans, various injuries, e.g. due to fast blunt impacts, dynamic and quasi-static clamping, or cuts by sharp tools may occur. In order to assemble a larger picture of this problem, we discussed and analyzed various worst-case scenarios in pHRI according to the following scheme

1) Select and/or define and classify the impact type

2) Select the appropriate injury measure(s)

3) Evaluate the potential injury of the human

4) Quantify the influence of the relevant robot parameters

5) Evaluate the effectiveness of countermeasures for injury reduction and prevention

Attempts to investigate

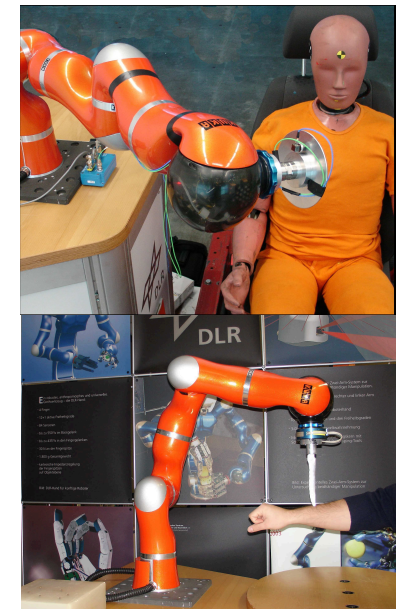

Fig. 3. Collision experiments with an LWR-III HIII dummy (upper) and human (lower).

pact tests at standardized crash-test facilities and to use the outcome to analyze safety issues during physical humanrobot interaction were carried out. In order to quantify the potential danger emanating from the LWR-III, impact tests at the Crash-Test Center of the German Automobile Club (ADAC) were conducted and evaluated, see Fig. 3 (upper). Consecutive work extended the initial analysis for various other robot types, clamping, and even to sharp contact, see Fig. 3 (lower). Generally, the analysis provides unique data that helps explaining the characteristics of robot-human impacts, which in turn can be used for safer robot design and control. Furthermore, the results are used as an input for future service robotics standards that define "safe" behavior of robots in human environments.

\section{Real-time motion planning}

Up to now, we discussed rather the design and lowlevel control schemes for our robots. However, the real-time 


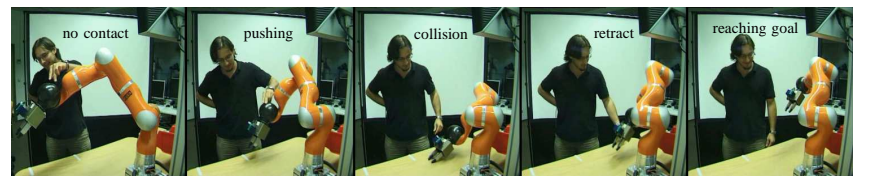

Fig. 4. Automatic recovery from physical collisions with real-time motion planning.
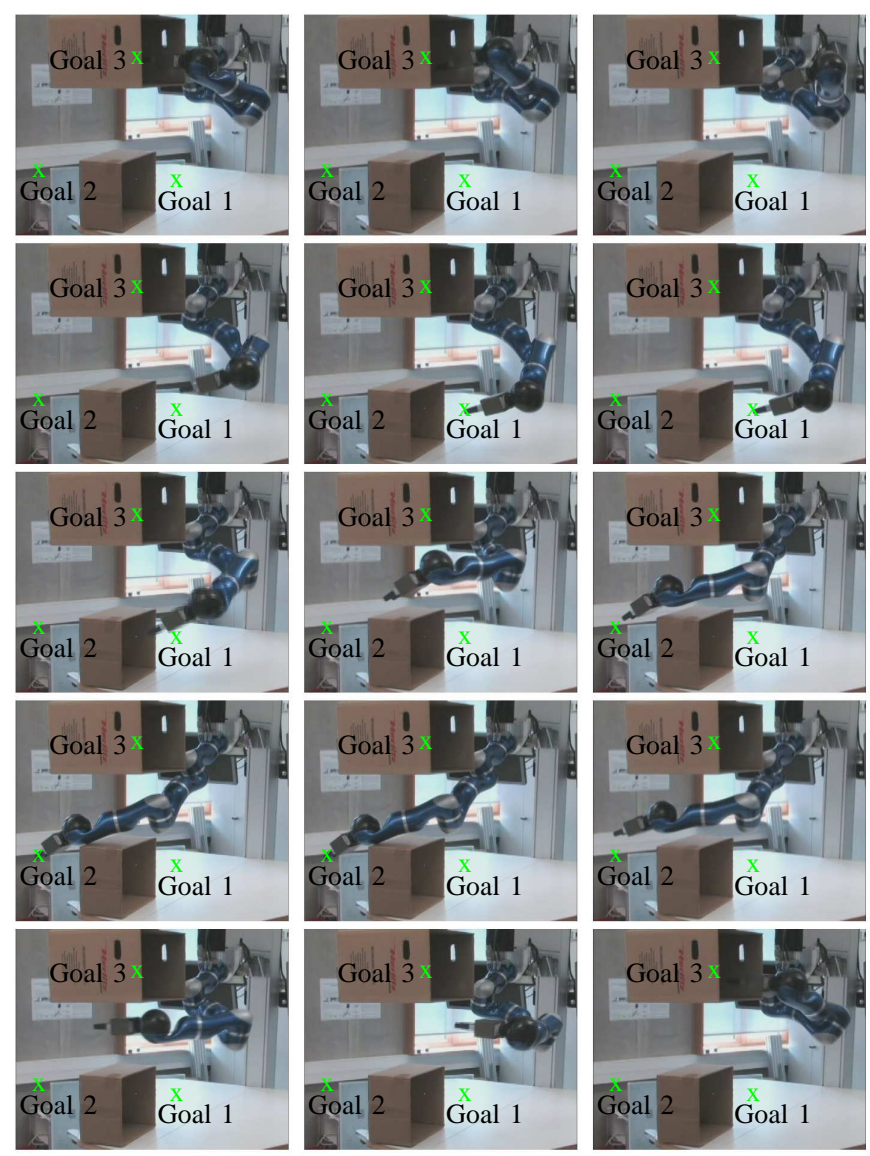

Fig. 5. Real-time motion planning at $500 \mathrm{~Hz}$ for a global 3-goals motion planning problem.

planning and execution of motions in a dynamic and partially unknown environment is fundamental for autonomous and safe acting. If contact is desired or inevitable, also motion planning should be able to robustly and safety handle it, see Fig. 4. However, typically this is only approached as a pure control problem. Nonetheless, we believe this to be a rather artificial separation that misses the chance of designing more sophisticated responses to contact on trajectory level as well. Especially pHRI is a field in which such behavior is certainly desired. As human and robot shall collaborate very closely, the problem of generating "human-friendly" motions is of large interest. We developed several methods for dealing with obstacles and contact in real-time [15], [16] on motion planning level. We could show for several problems, which were typically a domain for global sampling based planners that they can be solved in hard real-time ${ }^{3}$ with local methods only, see Fig. 5. This is due to the fact

${ }^{3}$ Our current implementation runs at $500 \mathrm{~Hz}$. Presumably, the high
parallelizability of our algorithm will enable us to further speed up the parallelizability of our algorithm will enable us to further speed up the scheme.

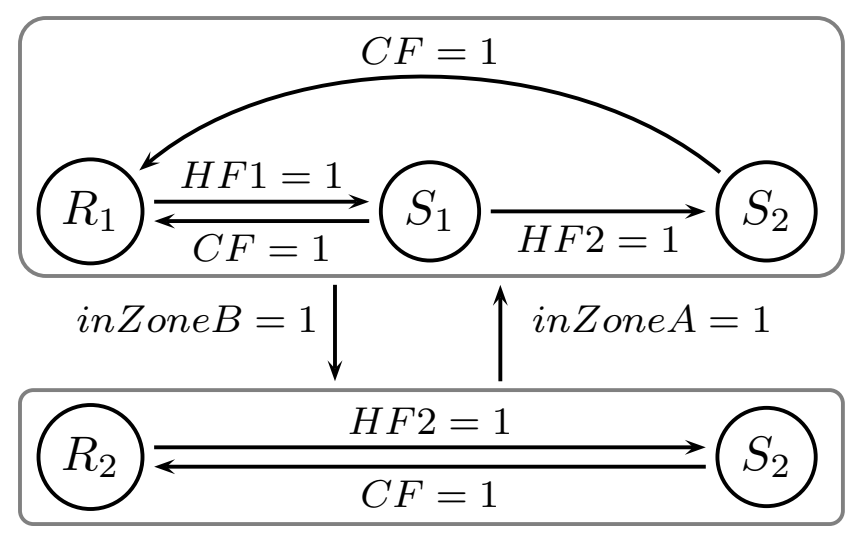

Fig. 6. Simple discrete planner for realizing context sensitive behavior of a robot. This example scheme enables the robot to behave differently during free motion and object manipulation phase. In this example $R_{1}, R_{2}$ are the nominal behaviors in zone $A$ and zone $B$, respectively. in $Z$ one $A$ and in $Z$ one $B$ indicate whether the robot is operating in free space or close to the object, defined by a encapsulated surface of certain maximum distance to the object. $S_{1}$ denotes the safety reflex behavior for stopping abruptly and $S_{2}$ for switching to torque control with gravity compensation. $C F$, $H F 1$, and $H F 2$ denote human confirmation and contact severity level.

that these algorithms have favorable convergence properties. Another key feature of these schemes is the unified treatment of virtual and physical forces, which allows the systematic fusion of obstacle avoidance with collision retraction or exploratory tactile behavior.

\section{E. Behavior based control for safe acting and manipulation}

Due to the diversity and complexity of the developed control capabilities and their sheer number it is non-trivial to design, implement and switch between them consistently under the premise of ensuring safe behavior. For that reason we developed a control architecture and a formal representation structure for interactive robots, which contains and consistently combines a wide set of strategies for safe manipulation and human-friendly behavior [25], [17]. We designed an encapsulated low-level control framework, which provides a discrete atomic action interface, which smallest primitive is defined as atomic action $:=$ (command, behavior). command can be e.g. atomic-move2, switch-behavior, or a simple stop. This is a rather classical approach. However, in contrast to other robots, the behavior is in our case a very complex data structure that defines the "overall" control activation the robot occupies. It defines a minimal representation of the activated interaction, motion, and local decision capabilities of the robot. This intuitive level of abstraction gives the task programmer or task planner a very powerful interface to the robot. Furthermore, we distinguish between operational behavior and reflex behavior.

- Operational behaviors: a formal high-level parametrization of the robot capabilities that defines its particular motion, control, and safety properties. This fully determines the nominal motion control and disturbance response of a robot. The atomic components of any general task automaton are operational behaviors.

- Reflex behaviors: a formal parameterization of a realtime reflex behavior of a robot that is associated with a real-time activation signal. This represents either the 
indication of a certain stimulus or a fault ${ }^{4}$. Reflexes override the currently active operational behavior and execute a low-level strategy. Complex reflex patterns are directed reflex graphs, which represent a decisional component in the inner most control loop.

Figure 6 depicts a simple example for illustrating the concept. Generally, the described approach intends to tightly couple the block world and control world, i.e. leaving the common separation based designs. The presented design is from our point of view a missing link between control and task planning for interactive robots.

\section{POTENTIAL IMPACT}

The potential impact of the presented work is manifold. First, the understanding of human injury in robotics is a novel research field that has created a worldwide community working on it. It forms an interdisciplinary complex involving robotics, biomechanics, and medicine. Furthermore, our results contribute to a basis for new service robotics standards that are currently being created for regulating acting and manipulation in human environments. Together with our work on physical Human-Robot Interaction in design, control, real-time motion planning, and real-time task planning, it seems that we are only a blink away from having first complex manipulation and interaction realworld scenarios. These would start from fundamentally new manufacturing processes with moderate interaction in the automobile sector to full scale pHRI tasks, incorporating dynamic interaction for complex processes. On this basis, we can also lay the ground to pursue real-world domestic applications that would heavily benefit from the experiences made in the industrial sectors. However, in both application areas one of the main concerns with respect to robots coming to everyday life is whether they could be able to harm humans. This is a factor that can significantly hinder the success of robotics in everyday life. In our research we take this concern very serious and even make it our central task.

\section{ACKNOWLEDGMENT}

This work has been partially funded by the European Commission's Sixth Framework Programme as part of the project VIACTORS under grant no. 231554 and SAPHARI under grant no. 287513.

\section{REFERENCES}

[1] A. Albu-Schäffer, S. Haddadin, C. Ott, A. Stemmer, T. Wimböck, and G. Hirzinger, "The DLR lightweight robot - lightweight design and soft robotics control concepts for robots in human environments," Industrial Robot Journal, vol. 34, no. 5, pp. 376-385, 2007.

[2] R. Bischoff, J. Kurth, G. Schreiber, R. Koeppe, A. Albu-Schäffer, A. Beyer, O. Eiberger, S. Haddadin, A. Stemmer, G. Grunwald, and G. Hirzinger, "The kuka-dlr lightweight robot arm: a new reference platform for robotics research and manufacturing," in International Symposium on Robotics (ISR2010), Munich, Germany, 2010.

[3] A. Albu-Schäffer, C. Ott, and G. Hirzinger, "A unified passivitybased control framework for position, torque and impedance control of flexible joint robots," The Int. J. of Robotics Research, vol. 26, pp. 23-39, 2007.

[4] S. Haddadin, A. Albu-Schäffer, A. D. Luca, and G. Hirzinger, "Collision detection \& reaction: A contribution to safe physical human-robot interaction," in IEEE/RSJ Int. Conf. on Intelligent Robots and Systems (IROS2008), Nice, France, 2008, pp. 3356-3363.

${ }^{4}$ Stimuli are general perception inputs, whereas faults are detected either by processed stimuli (observation of external torques, proximity information, ...) or general system malfunctions, as e.g. communication collapse or runtime violations.
[5] C. Ott, Cartesian Impedance Control of Redundant and Flexible-Joint Robots. Springer Publishing Company, Incorporated, 2008.

[6] A. Stemmer, A. Albu-Schäffer, and G. Hirzinger, "An analytical method for the planning of robust assembly tasks of complex shaped planar parts," in Int. Conf. on Robotics and Automation (ICRA2007), Rome, Italy, 2007, pp. 317 - 323.

[7] S. Haddadin, A. Albu-Schäffer, and G. Hirzinger, "Safety evaluation of physical human-robot interaction via crash-testing," Robotics: Science and Systems Conference (RSS2007), Atlanta, USA, pp. 217-224, 2007.

[8] — , "Dummy crash-tests for the evaluation of rigid human-robot impacts," in IARP International Workshop on Technical challenges and for dependable robots in Human environments (IARP2007), Rome, Italy, 2007.

[9] - "Requirements for safe robots: Measurements, analysis \& new insights," The Int. J. of Robotics Research, vol. 28, no. 11-12, pp. 1507-1527, 2009.

[10] S. Haddadin, A. Albu-Schäffer, M. Frommberger, J. Rossmann, and G. Hirzinger, "The "DLR Crash Report": Towards a standard crashtesting protocol for robot safety - part I: Results," in IEEE Int. Conf. on Robotics and Automation (ICRA2008), Kobe, Japan, 2009, pp. 272279.

[11] S. Haddadin, A. Albu-Schäffer, and G. Hirzinger, "Soft-tissue injury in robotics," IEEE Int. Conf. on Robotics and Automation (ICRA2010), Anchorage, Alaska, pp. 3462-3433, 2010.

[12] - "Soft-tissue injury caused by sharp tools: Definitions, experiments and countermeasures," accepted at: IEEE Robotics and Automation Mag., 2011

[13] J. Park, S. Haddadin, J. Song, and A. Albu-Schäffer, "Designing optimally safe robot surface properties for minimizing the stress characteristics of human-robot collisions," in IEEE Int. Conf. on Robotics and Automation (ICRA2011), Shanghai,China, 2011, pp. 5413-5420.

[14] A. De Luca, A. Albu-Schäffer, S. Haddadin, and G. Hirzinger, "Collision detection and safe reaction with the DLR-III lightweight manipulator arm," in IEEE/RSJ Int. Conf. on Intelligent Robots and Systems (IROS2006), Beijing, China, 2006, pp. 1623-1630.

[15] S. Haddadin, H. Urbanek, S. Parusel, D. Burschka, J. Roßmann, A. Albu-Schäffer, and G. Hirzinger, "Realtime reactive motion generation based on variable attractor dynamics and shaped velocities," in IEEE/RSJ Int. Conf. on Intelligent Robots and Systems (IROS2010), Taipeh, Taiwan, 2010, pp. 3109-3116.

[16] S. Haddadin, R. Belder, and A. Albu-Schäffer, "Dynamic motion planning for robots in partially unknown environments," in IFAC World Congress (IFAC2011), Milano, Italy, vol. 18, 2011.

[17] S. Parusel, S. Haddadin, and A. Albu-Sch, "Modular state-based behavior control for safe human-robot interaction : A lightweight control architecture for a lightweight robot," in IEEE Int. Conf. on Robotics and Automation (ICRA2011), Shanghai,China, 2011, pp. 4298-4305.

[18] J. Vogel, S. Haddadin, J. D. Simeral, D. Stavisky, S. D. Bacher, L. R. Hochberg, J. P. Donoghue, and P. van der Smagt, "Continuous control of the DLR Lightweight Robot III by a human with tetraplegia using the BrainGate2 neural interface system," in International Symposium on Experimental Robotics (ISER2010), Dehli, India, 2010.

[19] N. Hogan, "Impedance control: An approach to manipulation: Part I - theory, Part II - implementation, Part III - applications," Journal of Dynamic Systems, Measurement and Control, vol. 107, pp. 1-24, 1985 .

[20] F. B. Goldsmith, P. B. and A. Goldenberg, "Stability of hybrid position/force control applied to manipulators with flexible joints," Int. Journal of Robotics and Automation, no. 14, pp. 146-159, 1999.

[21] A. Albu-Schäffer, C. Ott, and G. Hirzinger, "A passivity based cartesian impedance controller for flexible joint robots - Part II: Full state feedback, impedance design and experiments," in Int. Conf. on Robotics and Automation (ICRA2004), New Orlenas, USA, 2004, pp. 2666-2673.

[22] L. Zollo, B. Siciliano, A. De Luca, E. Guglielmelli, and P. Dario, "Compliance control for an anthropomorphic robot with elastic joints: Theory and experiments." ASME Journal of Dynamic Systems, Measurements and Control, no. 127, pp. 321-328, 2005.

[23] J. Craig and M. Raibert, "A systematic method for hybrid position/force control of a manipulator," IEEE Computer Software Applications Conf., pp. 446-451, 1979.

[24] C. Yang, G. Gowrishankar, S. Haddadin, S. Parusel, A. Albu-Schäffer, and E. Burdet, "Human like adaptation of force and impedance in stable and unstable interactions," accepted: IEEE Transactions on Robotics, 2011.

[25] S. Haddadin, M. Suppa, S. Fuchs, T. Bodenmüller, A. Albu-Schäffer, and G. Hirzinger, "Towards the robotic co-worker," in International Symposium on Robotics Research (ISRR2009), Lucerne, Switzerland, 2009. 